\title{
Advanced Favorable Hodgkin Lymphoma
}

National Cancer Institute

\section{Source}

National Cancer Institute. Advanced Favorable Hodgkin Lymphoma. NCI Thesaurus.

Code C68662.

An advanced Hodgkin lymphoma with favorable prognosis. 